\title{
The Low FODMAP Diet and Its Application in East and Southeast Asia
}

\author{
Marina lacovou, ${ }^{1 *}$ Victoria Tan, ${ }^{2}$ Jane G Muir, ${ }^{1}$ and Peter R Gibson ${ }^{1}$ \\ ${ }^{1}$ Department of Gastroenterology, Monash University and Alfred Hospital, Melbourne, Australia; and ${ }^{2}$ Department of Medicine, University of \\ Hong Kong, Hong Kong
}

There is growing interest in using food choice/dietary change to influence clinical outcomes in patients with irritable bowel syndrome (IBS). The low fermentable oligo-, di-, mono-saccharides, and polyols (FODMAPs) diet is an evidence-based approach that is gaining popularity in many Western countries. The low FODMAP diet is based on restricting dietary intake of short chain carbohydrates that are slowly absorbed or indigestible and not absorbed during passage through the small intestine. These are collectively described as "FODMAPs" and comprise oligosaccharides (mostly fructans, galacto-oligosaccharides), sugar polyols, fructose in excess of glucose, and lactose in lactose malabsorbers. The general strategy of the diet is to avoid foods high in FODMAPs and replace them with foods low in FODMAPs, with long-term restriction limited to what is required to control symptoms. The likely mechanism of action is minimisation of the stimulation of mechanoreceptors exerted by distension of the intestinal lumen with water from osmotic effects and gases from bacterial fermentation in those with visceral hypersensitivity. The success of this dietary approach greatly depends on detailed knowledge about the FODMAP composition of food commonly consumed in that country. While the content of foods associated with East and Southeast Asian cuisines has not been fully explored, major high FODMAP sources are frequently used and include onion, garlic, shallots, legumes/pulses, and wheat-based products. Thus, this dietary approach holds great promise in treating IBS patients in East and Southeast Asia. The aim of this review is to highlight how the diet is implemented, its efficacy, and troublesome ingredients frequently used in Asian dishes.

(J Neurogastroenterol Motil 2015;21:459-470)

Key Words

Asia; Diet; FODMAPs; Irritable bowel syndrome

\section{Introduction}

The epidemiology of functional gastrointestinal disorders (FGID) varies across countries and cultures, but irritable bowel syndrome (IBS) appears to be a global phenomenon. ${ }^{1,2}$ Prevalence varies according to the criteria used for diagnosis, but in East and Southeast Asia prevalence varies across countries, from South Korea 6-15\%, China 2-10\%, Japan 6-14\% to Thailand 6\%. ${ }^{3}$ Prevalence is in general lower than in Western countries,

Received: June 27, 2015 Revised: July 29, 2015 Accepted: August 9, 2015

(c) This is an Open Access article distributed under the terms of the Creative Commons Attribution Non-Commercial License (http://creativecommons. org/licenses/by-nc/4.0) which permits unrestricted non-commercial use, distribution, and reproduction in any medium, provided the original work is properly cited.

${ }^{*}$ Correspondence: Marina lacovou, Ms

Level 6/99 Commercial Rd, Melbourne 3004, Australia

Fax: +61-399030392, E-mail: marina.iacovou@monash.edu

Financial support: Marina lacovou is funded by Monash University, Australian Postgraduate Award (APA).

Conflicts of interest: None.

Author contributions: Marina lacovou contributed to the conception, writing, editing, and approval of the manuscript; Victoria Tan contributed to the writing, editing, and approval of the manuscript; Jane G Muir contributed to the conception, editing, and approval of the manuscript; and Peter R Gibson contributed to the conception, writing, editing, and approval of the manuscript.

ORCID: Marina lacovou, http://orcid.org/0000-0001-5209-0624. 
but, at least in Korea, IBS appears to be increasing at an annual rate of $1 \%{ }^{4}$ Globally, there is a consistent female predominance, but Asia-specific studies have found that the prevalence of IBS has no significant sex difference. ${ }^{5-8}$

In alignment with the multiple apparent contributing pathogenic factors, management of IBS is multi-modal and includes pharmacological, neuromodulatory, dietary, psychological and other behavioral approaches. In Western countries such as those in North America, Australia, New Zealand, and Western Europe, there has been an increasing interest in utilising diet as a primary treatment modality rather than as an adjunct to pharmacotherapy. While many diets have been described and applied, the evidence-base for most is poor. However, a diet that reduces the intake of foods containing indigestible and slowly absorbed short-chain carbohydrates, collectively termed "FODMAPs" (fermentable oligo-, di-, mono-saccharides, and polyols), has a growing number of studies that consistently describe efficacy in about $70 \%$ of an unselected population of patients with IBS (Table). ${ }^{9-12}$ As a result, the low FODMAP diet is emerging as a first line of treatment for IBS sufferers.

The low FODMAP diet, however, varies markedly across geographical regions. The current database of food content specifically addressing FODMAPs is predominantly from Australia and, to a lesser degree, North America, United Kingdom, and Scandinavian countries. Even though the traditional dietary patterns of many Asian cultures are likely to change along with rapid economic growth in Asia and the introduction of Western culture and food consumption patterns, ${ }^{1,2}$ this raises obvious questions about the applicability of the diet to Chinese, Korean, or Japanese cuisine and the FODMAP content of food eaten in those regions. This current review will address the nature of the low FODMAP diet and the evidence for efficacy, and examine its relevance to countries in the dietary cuisine of East and Southeast Asia.

\section{The Low FODMAP Diet}

\section{FODMAPs}

FODMAPs are naturally found in a wide range of foods. They are a group of fermentable short-chain carbohydrates that are either indigestible in the gut due to the absence of appropriate hydrolases, or slowly absorbed in the small intestine. They comprise: indigestible "oligosaccharides" that include fructo-oligosaccharides (fructans), found in garlic, onion, wheat, barley and rye, and galacto-oligosaccharides (GOS), found in legumes, nuts, soy beans, and soy products; "lactose" (a disaccharide that will not be absorbed if undigested by lactase) found in milk and yoghurt; "fructose" (a monosaccharide) when in excess of glucose, found in certain fruits such as apple, pear, mango, watermelon, tamarillo, and honey; and "polyols" (or sugar alcohols) that include sorbitol found in apricots, avocado and lychee, and mannitol found in mushrooms, snow peas, and cauliflower. The physiology of their absorption patterns and/or digestion has been reviewed in detail by Tuck et al. ${ }^{13}$

There are other short-chain carbohydrates that might act as FODMAPs. Isomalto-oligosaccharides (IMO) are partly hydolyzed although substantial proportion does enter the colon. ${ }^{14,15}$ IMO are present in honey and, according to Japanese literature, some fermented foods such as miso, soy sauce, and saki, ${ }^{14,16}$ but the actual concentrations are uncertain. IMO may also be added as a prebiotic/fiber to some food. Another class of oligosaccharides, the xylo-oligosaccharides (XOS), do not seem to be naturally present in food, but they can be derived from arabinoxylans and are being explored as prebiotics to add to food.

\section{Physiological Effects of FODMAPs}

FODMAPs, being small water-soluble molecules that are poorly absorbed from the intestinal lumen, are osmotically active through the bowel. By attracting more water into the lumen of the small bowel, as elegantly demonstrated by magnetic resonance imaging studies, ${ }^{17,18}$ they cause luminal distension and will deliver more water to the colon. ${ }^{19}$ Once FODMAPs reach the large bowel, they are fermented by intestinal bacteria, resulting in the release of gases (hydrogen, carbon dioxide, and methane) which leads to luminal distension of the large bowel. These effects will occur in all humans consuming FODMAPs and, under normal circumstances, would not cause more than minor bloating or discomfort at worst. However, those who have visceral hypersensitivity and/or abnormal motility responses to luminal distension are then at risk of developing symptoms associated with IBS, including abdominal pain and bloating, flatulence and constipation, ${ }^{9,11,13}$ by virtue of stimulation of mechanoreceptors.

Another very important attribute associated with bacterial fermentation of unabsorbed carbohydrates is the production of short-chain fatty acids (SCFA), particularly acetate, propionate and butyrate, which exert a wide range of effects, both beneficial and detrimental. ${ }^{20}$ SCFA can promote water and sodium absorption and in this way reduce the risk of osmotic diarrhoea. ${ }^{20}$ Butyrate, in particular, is the most important energy source of the 


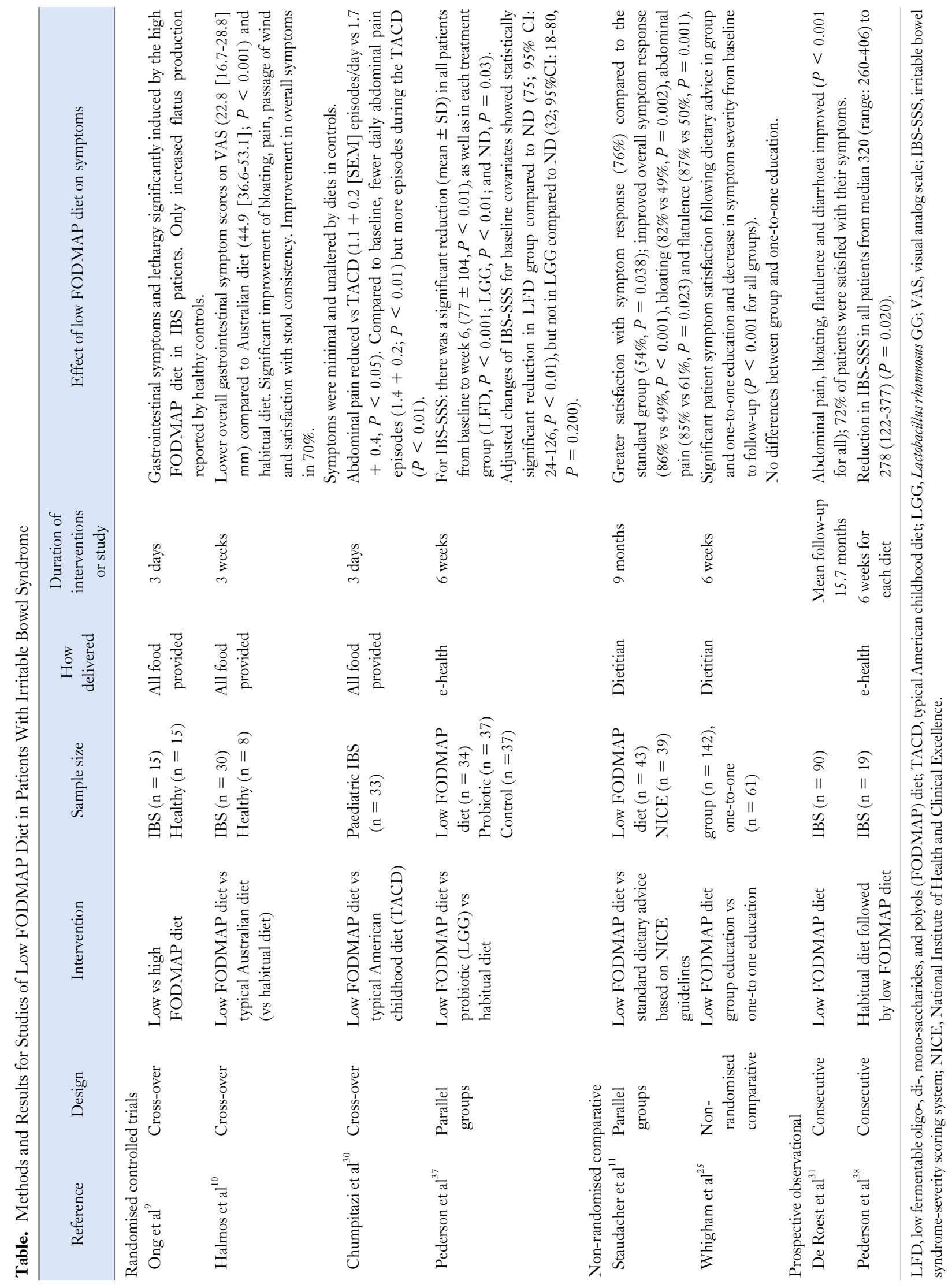


colonic epithelium and has a range of effects relevant to the health and maintenance of gut epithelial health. ${ }^{21,22}$ Propionate and acetate may also have systemic immunomodulatory and epigenetic effects. On the negative side, butyrate can increase visceral sensitivity and all the SCFA can be toxic to epithelium if present in high concentrations.

Indigestible oligosaccharides (fructans and GOS) also alter the growth characteristics of certain resident colonic bacteria, the so-called prebiotic effect. The ingestion of supplemental FOS, GOS, IMO, or XOS will selectively lead to increased relative abundance of bacteria of presumed health-promoting properties. ${ }^{20,21,23}$ Such effects have now been shown for diets that vary in the amount of naturally-occurring oligosaccharides ingested. In a randomised-controlled trial, 19 patients with IBS who followed a dietitian-delivered low FODMAP diet for 4 weeks had a significant reduction in relative abundance in the feces of bifidobacteria at follow-up compared with that of 22 patients who followed their habitual diet. ${ }^{12}$ In a randomised blinded cross-over study involving 27 patients with IBS and 6 healthy subjects, fecal microbiota were characterised in association with habitual and the 2 study diets that were low or moderate in FODMAP content. Lowering FODMAP intake clearly reduced the relative abundance of all bacteria. However, the relative abundance of beneficial butyrate-producing bacteria of the Clostridium cluster XIVa and of the mucin-degrading bacterium, Akkermansia muciniphila were increased, and the relative abundance of unfavorable mucus-consuming bacterium, Ruminococcus torques, had decreased in the feces of subjects consuming the higher, controlled FODMAP diet compared with those in both the habitual and low FODMAP diets. ${ }^{24}$ These observations raised issues regarding implications of strict and long-standing FODMAP restriction, but, more importantly, highlighted what had previously been ignored - the importance of natural prebiotics in the diet.

Increasing the FODMAP intake can induce tiredness in patients with IBS and aggravate gastroesophageal reflux in healthy adults and those with gastro-esophageal reflux disease. Mechanisms by which these associations occur have not been satisfactorily defined, but may involve secondary changes in circulating mediators. More work is needed to define additional physiological and pathophysiological effects of consuming FODMAPs.

\section{Implementation of the Low FODMAP Diet}

The principles of the low FODMAP diet are that the patients avoids all foods with high FODMAP content in normal serving sizes and replaces them with those low in FODMAPs. This "exchange" is important as FODMAPs are found in multiple food groups and ensures patients consume foods from all of the 5 core food groups: dairy, meat and meat alternatives, fruit, vegetables and legumes, and grains and cereals. Recommended low FODMAP serving sizes are based on the Australian Guide to Healthy Eating guidelines, to ensure that a patient's optimal nutrition is not compromised. The low FODMAP diet is typically taught by a qualified dietitian with expertise in the area of gastrointestinal disorders, once a diagnosis of IBS has been made by experts, such as gastroenterologists. Dietitian-patient consultations are usually managed using one-on-one meetings, but group consultations may also be equally effective and more cost-effective. ${ }^{25}$ A strict low FODMAP diet is generally prescribed by a dietitian for 2-6 weeks after which a steady re-introduction phase is followed, and closely monitored, until a balance between tolerated doses and symptom control is achieved. Dietitians in this area have the expertise not only to educate patients on which foods to consume and those to avoid, but also how to add flavor to their foods and how to interpret food labels/ingredients lists. They are also able to provide recipe ideas.

A key to the diet's successful implementation is knowledge of the FODMAP content of usual serving sizes of foods. There are many lists available on the internet, but many are inaccurate and out-dated. The Monash University Low FODMAP diet App ${ }^{26}$ and the Monash University Low FODMAP diet booklet ${ }^{27}$ are 2 resources developed in an attempt to circumvent these inaccuracies, since they are based entirely on measured food content and are regularly updated. They are offered by dietitians to patients to support their management of the diet. Both resources contain a comprehensive list of foods, beverages, and condiments that can be consumed while on a low FODMAP diet, including how to read labels, a dietary fiber counter and a menu guide with recipes.

There is no standard protocol when applying re-introduction techniques of higher FODMAP containing foods. Reintroduction techniques may be individualised to a patient's symptoms, food likes and dislikes, or what they are missing the most from their diet. Alternatively, a dietitian may introduce less troublesome FODMAPs to a patient's diet first such as polyol-containing foods, followed by lactose or fructose (in excess of glucose) containing foods, followed by fructans and/or GOS containing foods. This will vary from one individual to another. Regardless of which approach is taken, one food item is generally re-introduced at a time, every few days, until an achievable dose 
level is identified whereby symptoms are manageable and controlled.

It is important that patients work with a dietitian to help identify "tolerance levels" while ensuring that their diet is balanced, contains adequate dietary fiber, is nutritionally adequate and is not overly restricted. While FODMAP tolerances vary between individuals, it may mean that IBS patients eat high FODMAP foods less often or in smaller quantities to maintain a balance between the diet and IBS symptoms.

\section{Efficacy of the Low FODMAP Diet}

The low FODMAP dietary therapy to treat IBS has attracted worldwide attention since the publication of high quality studies to support its efficacy in $>70 \%$ of individuals. Experience of the diet is growing in many centres. ${ }^{10,13,25}$ Details of the types of studies and their specific results are shown in the Table. Such studies as well as those published in abstract form were subjected to a recent meta-analysis and systematic review, with the conclusion that the diet is effective in patients with IBS. ${ }^{28}$ The outcomes of such studies have also prompted the increasing need to translate the diet for practical use by other countries. ${ }^{25}$

The greatest improvement in overall gastrointestinal symptoms appears to occur within 7 days of adhering to a low FODMAP diet, after which symptoms remain relatively constant. ${ }^{10,29,30}$ This rapid improvement is likely to be the result of osmotic and motility changes in the gut. ${ }^{9}$ The durability of such benefits have been suggested in a prospective observational study, ${ }^{31}$ but further study of longer term effects are warranted. Adverse effects of the low FODMAP diet in the short-term have not been identified. However, 2 studies demonstrated that a low FODMAP diet significantly changes the composition of luminal bacteria to a putatively less health-promoting structure. ${ }^{10,12}$ The use of a pre- or probiotic while following the low FODMAP diet in the long-term has been suggested, but there are no data to support such an idea. ${ }^{12}$ Additionally, the low FODMAP diet may reduce an individual's dietary fiber intake if not properly followed, and calcium intake can be reduced if lactose is restricted. ${ }^{12}$ If the principles of the diet are followed and foods taken out of the diet are replaced by low FODMAP foods within the same groups and alternative fiber-containing foods are used, the diet should not impact nutritional adequacy. Studies to confirm this belief are needed.

In countries such as Australia and United Kingdom, dietetic support in the management of IBS using the low FODMAP diet is strong, and is now growing across United States and other parts of Europe. In East and Southeast Asia, health professionals may lack the knowledge to deliver such therapy in practice, but education resources are already developed and can be translated to suit Asian communities. The low FODMAP diet holds promise for good clinical outcomes for patients in Asia.

\section{Management of Irritable Bowel Syndrome in East and Southeast Asia}

The latest iteration of the Asia-Pacific Consensus statements for IBS state that the current focus of treatment is symptom relief and improvement in quality of life. ${ }^{6,32}$ Treatments in the AsiaPacific region are similar to those in the Western countries with the emphasis on a multi-disciplinary approach inclusive of a quality doctor-patient relationship, pharmacotherapy, psychiatrists on an as-needed basis, dietary modification and use of cognitive behavioral therapy and hypnotherapy. Various pharmacotherapy combinations are recommended, including, antispasmodic, laxative, prokinetic, antidiarrheal, anxiolytic/antidepressant, fiber, and antibiotic and probiotic agents. ${ }^{6}$ The current IBS consensus also acknowledges that food is commonly cited by subjects as a trigger to their symptomatology ${ }^{33,34}$ and that dietary restriction of certain "trigger" foods appears to improve symptoms. The current IBS consensus suggests the use of a patient food diary to identify trigger foods. However, no one specific diet has been recommended for subjects in Asia-Pacific for IBS. Furthermore, there is no specific recommendation to involve dietitians in the management of IBS in this region. The main recommendations at present include avoidance of excessive dairy products, chilli, curries, dietary fiber and excess fructose/fructo-oligosaccharide intake if they appear to be the cause of symptoms, with an emphasis on maintaining a nutritionally balanced diet.

\section{Translation of the Low FODMAP Diet to East and Southeast Asia}

\section{Current Food Lists of FODMAP Content}

The success of the low FODMAP diet has been largely the result of detailed knowledge of the FODMAP content of foods and ingredients. As outlined above, those high in FODMAPs can then be replaced with those low in FODMAPs within the same food group, leading to a lower risk of nutritional compromise. Accurate information about the FODMAP content of foods requires detailed analysis using well established techniques. ${ }^{35,36}$ 
Many foods to date have been tested for their FODMAP composition. Some of these foods are common to both Western and Asian cultures (Fig. 1-5). The ratings of an extensive list of foods, ingredients and beverages that have been tested for their FODMAP content are made available via The Monash University Low FODMAP Diet App as outlined above.
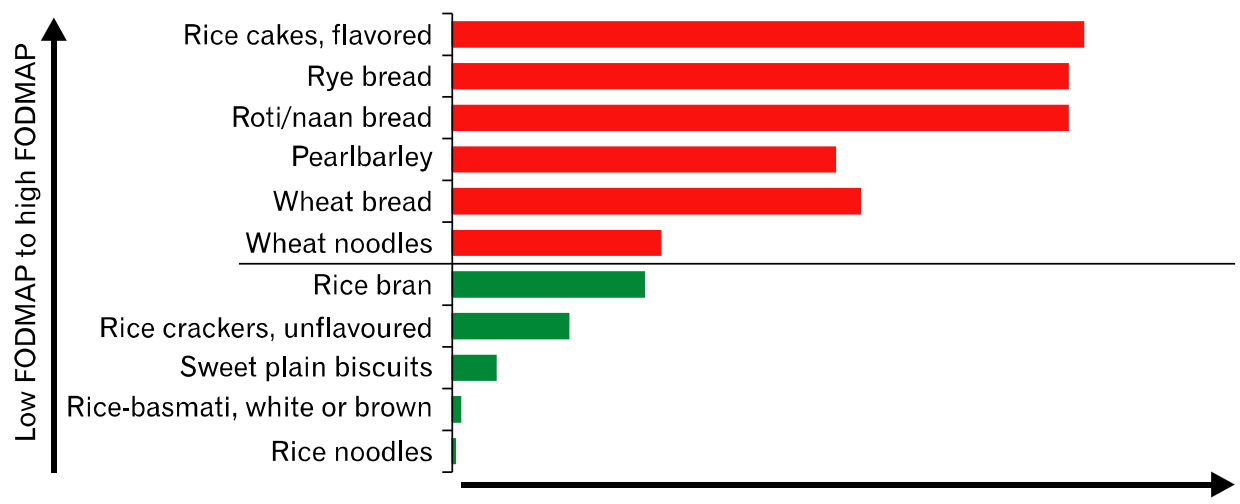

Low FODMAP to high FODMAP

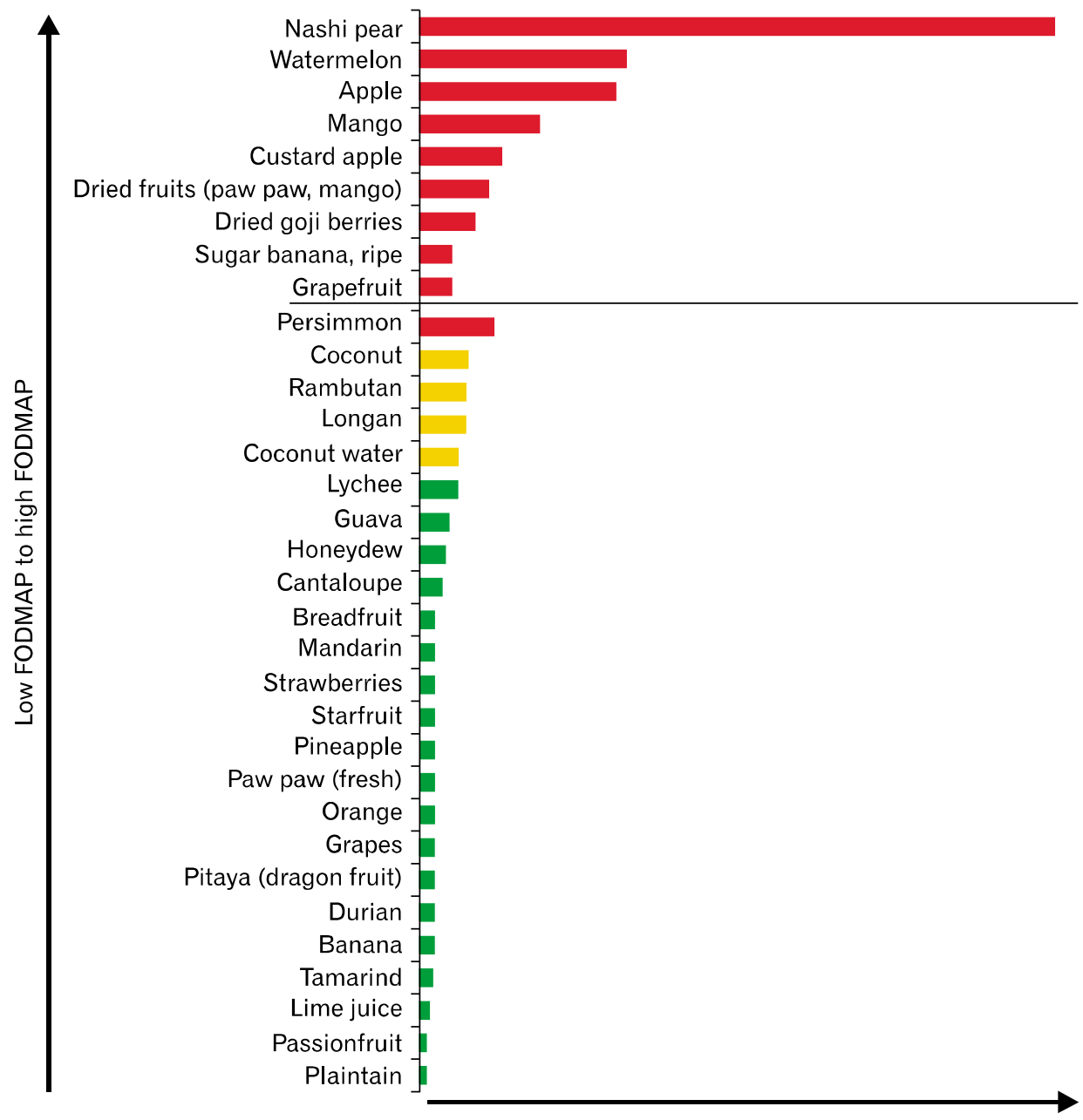

Low FODMAP to high FODMAP
Figure 1. Relative fermentable oligo-, di-, mono-saccharides, and polyols (FODMAPs) ratings of common cereals and grains (based on a typical serve). A relative FODMAP rating is given to each ingredient tested for its FODMAP content. For each FODMAP sub-unit, fructose, lactose, fructan, galacto-oligosaccharides, and polyols, there is variability of tolerated dose levels and therefore classifying them semi-quantitatively in acceptable serving sizes as low (in green), moderate (in amber), and high (in red) is a practical approach to support the implementation and management of the diet in clinical practice.

Figure 2. Relative fermentable oligo-, di-, mono-saccharides, and polyols (FODMAPs) ratings of common Asian fruits (based on a typical serve). For each FODMAP sub-unit, fructose, lactose, fructan, galacto-oligosaccharides, and polyols, there is variability of tolerated dose levels and therefore classifying them semi-quantitatively in acceptable serving sizes as low (in green), moderate (in amber), and high (in red) is a practical approach to support the implementation and management of the diet in clinical practice. 


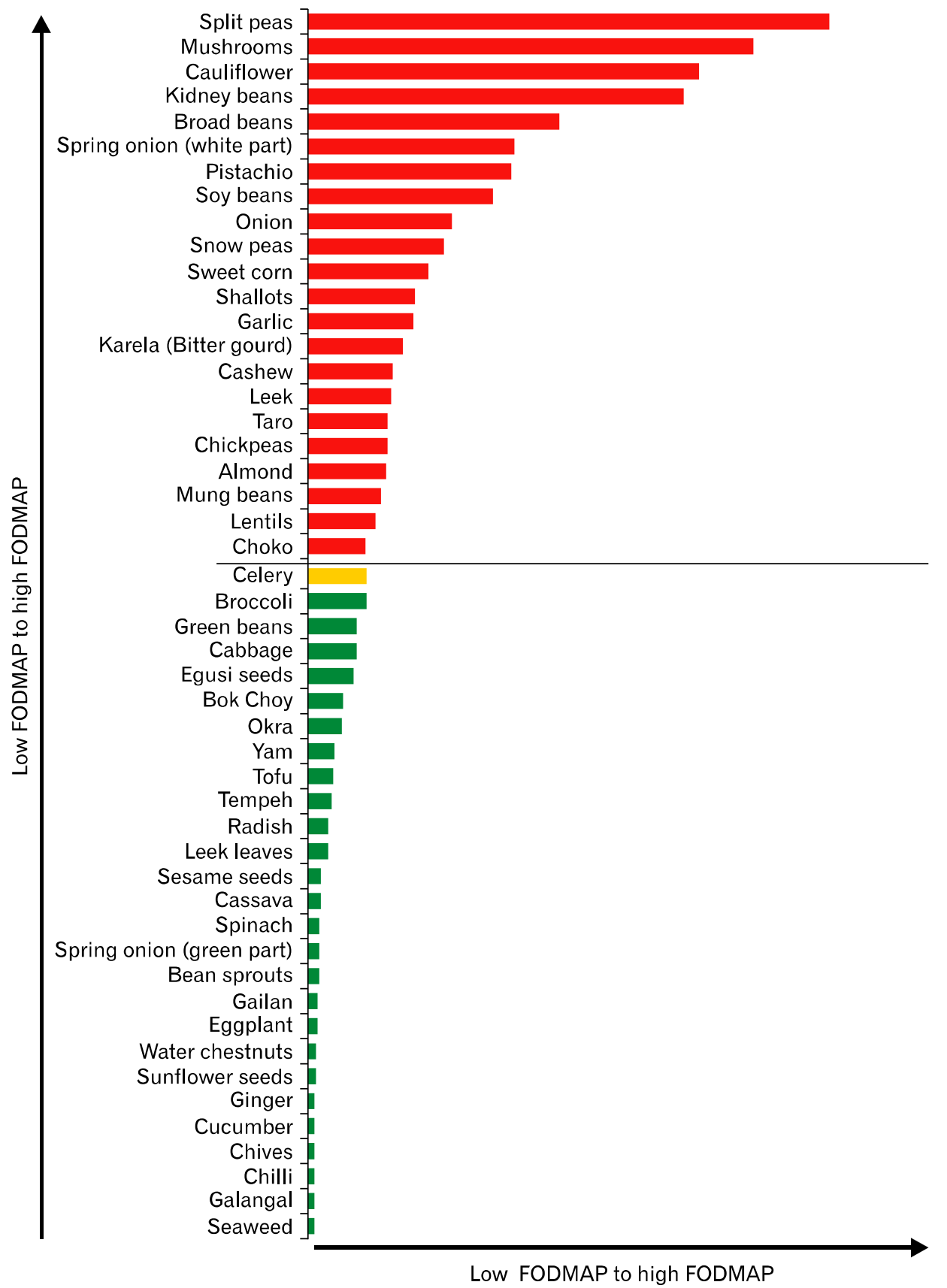

Figure 3. Fermentable oligo-, di-, monosaccharides, and polyols (FODMAPs) ratings of common Asian vegetables, tofu, legumes and nuts (based on a typical serve). Two examples where FODMAP content of foods/meals can change 1), although broccoli is rated low (green) for one serve (one-half of a cup or $47 \mathrm{~g}$ ), if consumed in larger quantities, eg, 1 cup, this increases the FODMAP content in one sitting giving it a high (red) FODMAP rating. 2) As with celery, when the serving size of one-half a medium stalk or $19 \mathrm{~g}$ is consumed in larger quantities during one sitting, the FODMAP rating will change to high.
However, difficulties arise in countries other than Australia. Not all foods used are available in Australia and those unique to regions may not have been tested. Furthermore, different names are used for similar products in different countries. For example, the salad vegetable, "rocket," is known as "arugula" in USA, and "rucola," in Europe. Cooking and preserving techniques might alter the content of FODMAPs in a specific food type. For example, cooking of legumes whereby the liquid component is dis- carded for the purpose of adding into a salad should theoretically reduce the GOS content as this is water soluble and would leach out. This would contrast with slow cooking of lentils in a curry, whereby the liquid component and its content of GOS is retained. Such ideas do require direct evaluation. Additionally, some foods with high FODMAP content might be eaten in small amounts in one country and, therefore, create few problems, but might form a larger proportion of the cuisine elsewhere. An example might be 

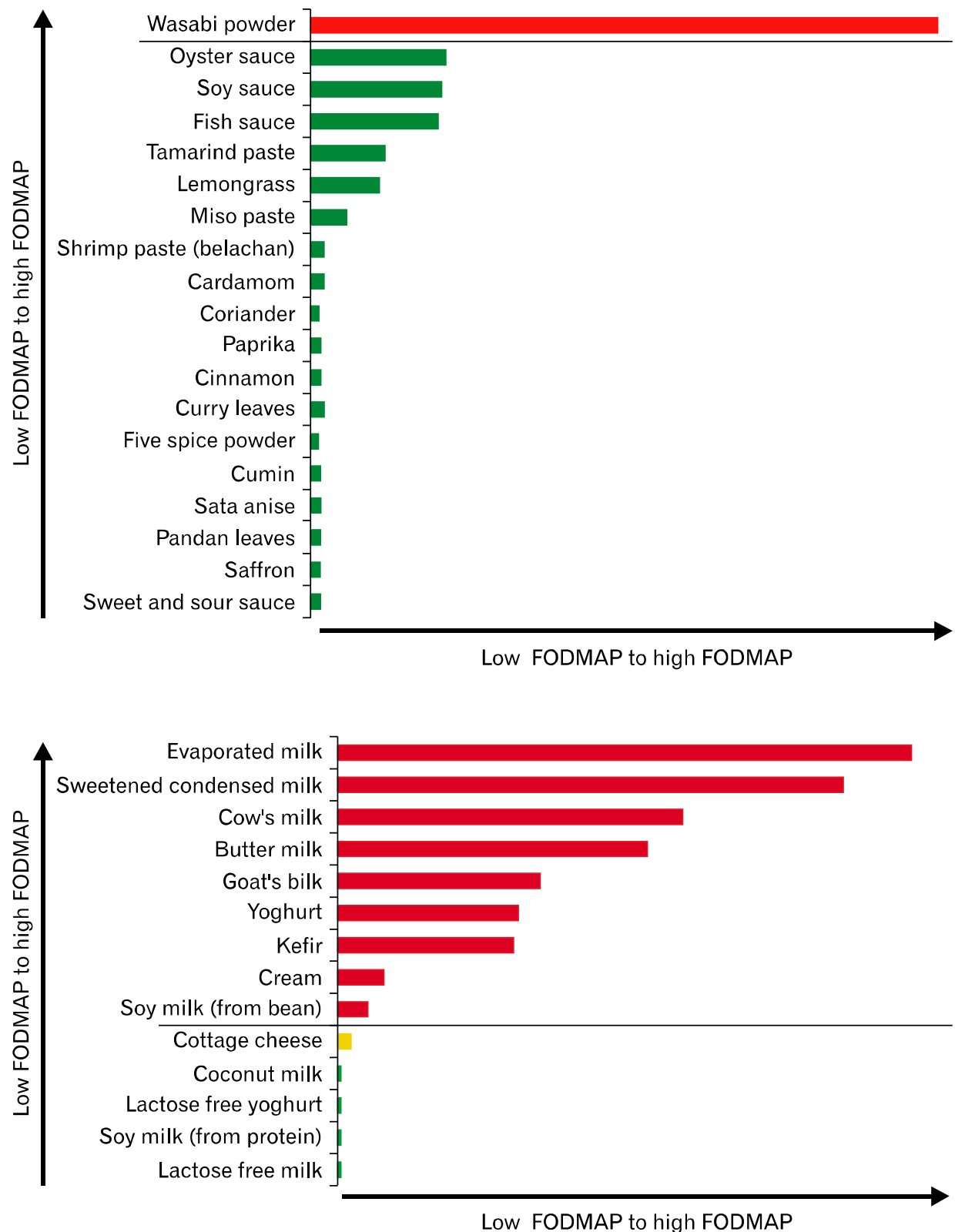

Figure 4. Relative fermentable oligo-, di-, mono-saccharides, and polyols (FODMAPs) ratings of commonly used condiments, herbs and spices. For each FODMAP sub-unit, fructose, lactose, fructan, galacto-oligosaccharides, and polyols, there is variability of tolerated dose levels and therefore classifying them semi-quantitatively in acceptable serving sizes as low (in green) and high (in red) is a practical approach to support the implementation and management of the diet in clinical practice.

Figure 5. Relative fermentable oligo-, di-, mono-saccharides, and polyols (FODMAPs) ratings of dairy products (based on a typical serve). For each FODMAP sub-unit, fructose, lactose, fructan, galacto-oligosaccharides, and polyols, there is variability of tolerated dose levels and therefore classifying them semi-quantitatively in acceptable serving sizes as low (in green), moderate (in amber), and high (in red) is a practical approach to support the implementation and management of the diet in clinical practice.

the use of garlic in Mediterranean countries in comparison to Northern Europe. Hence, in order to optimally implement the low FODMAP diet into other countries and cultures, knowledge of the FODMAP content of ingredients used and the way they are cooked and eaten are needed.

\section{FODMAPs in East and Southeast Asia}

Work in assessing many region-specific foods is in its infancy. However, since the low FODMAP diet is about "reducing the dose" of FODMAPs consumed over a day to achieve symptom control rather than "eliminating" FODMAPs from the diet, low
FODMAP strategies can still be constructed on current knowledge of food composition.

\section{Popular Foods in Asia With Known FODMAP Content}

There are many foods and ingredients that are used commonly across cultures and make up a clinically relevant proportion of most diets. Onions and garlic are 2 ingredients high in FODMAPs that are popular world-wide. Other popular ingredients used in many Asian dishes include high FODMAP vegetables such as cabbage, cauliflower, snow peas, mushrooms, 
leek and shallots. Many Asian countries are also adopting some more Western dietary habits, such as the inclusion of lactose-containing milk and dairy products. However, alternatives to these high FODMAP ingredients have already been identified as ideal substitutes to maintain the integrity of traditional dishes. For instance, the green part of spring onion and whole chives can been used in place of white onion and garlic respectively. Also garlic-infused oils may be used in dishes, once the garlic has been discarded. Lactose-free dairy products are widely available in place of regular lactose-containing products such as milk and yoghurt, or alternatively an oral lactase enzyme may be taken with the ingestion of lactose-containing products.

The cuisines of many East and Southeast Asian countries have particular characteristics that potentially might contribute to FODMAP intake. Several examples are outlined below where confidence can be drawn in terms of the likely FODMAP content of traditional dishes.

\section{Korean Dishes}

"Kimchi," a traditional Korean side dish customarily made from napa cabbage (Chinese cabbage), fermented and pickled with sweet, sour or spicy ingredients and garlic, is widely used in Korea and acts as an accompaniment to most meals. Kimchi may also be made from other fermented vegetables that might include radish, scallion (green onion or spring onion) or cucumber. "Doenjang," a fermented bean paste traditionally homemade with soy beans and brine, is used as a condiment or to flavor foods. Food companies, however, are known to include wheat flour in the manufacturing of this product. Doenjang is commonly mixed with garlic, onion, "gochujang" (a condiment made from red chilli, glutinous rice and fermented soybeans) and sesame oil, to make a thick, spicy paste called "ssamjang." Ssamjang is then served with grilled meats or a dish called ssambap (rice wrapped in leaf vegetables). Doenjang, gochujang and ssamjang collectively contain high FODMAP ingredients such as garlic, onion, soy bean and, in some cases, wheat flour. While kimchi, doenjang, gochujang, and ssamjang have several high FODMAP-containing ingredients and are likely to be on the high FODMAP list, the final product that is eaten in its usual serve size requires evaluation.

\section{Japanese Dishes}

In Japanese cuisine, aside from fish and sushi, noodles and gyozas are also common features. Both are generally made from dough using wheat flour, a high FODMAP ingredient. "Gyozas" are typically filled with a minced meat (eg, pork, beef, chicken, fish, or shrimp) and mixed with chopped vegetables. The vegetables that are popular in the filling mixture include cabbage, scallion (spring onion), leek and garlic, all of which are high FODMAP ingredients. Dumplings, the equivalent of gyozas, are also common in other Asian countries, including China ("wontom" and "dim sum") and Korea ("mandu”). Wontons comprise a dough shell that is filled with meat or mixed vegetables predominantly cabbage or chives with garlic and onion. Wontons are often eaten as a side dish or in a broth-type soup with or without noodles in the broth. It would be surprising if the final product is not high in FODMAP content given its ingredients.

\section{Thai Dishes}

Though typically accompanied by rice, Thai dishes also consist of noodle-based meals and clear broth-type soups with wontons. Other meals traditional to Thailand include "Tom Yum soup," a spicy clear soup mixed with chicken, prawns or mushrooms and garlic. Curries and stews, which may contain a number of high FODMAP vegetables (such as onion, cabbage, and garlic), are often consumed together with an array of condiments that contain garlic, are frequently used to compliment any meal or are used as a dipping sauce. Curry paste itself often contains high FODMAP ingredients that might include garlic (high FODMAP), shrimp paste (low), shallots (high), green/red chillies (low), coriander leaves (low), soy beans (high), and other herbs and spices. The finished product would likely be high in FODMAPs per gram, but whether they would confer at-risk high levels of FODMAPs to the curry would depend upon how much of the paste is used. Thus, evaluation of this is required to more accurately make specific advice.

\section{Chinese and Hong Kong Dishes}

In China and Hong Kong, condiments are commonly consumed, similar to Korean and other Asian cuisines, and are often made up with high FODMAP ingredients such as garlic, onions, shallots and honey. These condiments typically accompany traditional dishes that also frequently include high FODMAP-containing vegetables, such as, mushrooms, leeks, cabbage (both fresh and preserved) and legumes/pulses such as soya and mung beans. Rice and wheat are the 2 most common grains consumed. Whilst rice is an ingredient very low in FODMAPs, wheat is deemed high (fructans) and consumed in the form of wontons (as described earlier), "man tou" (unfilled wheat-based buns), "bao" (steamed wheat buns with a savoury or sweet filing) and noodles. 
Sweet soups common in China and Hong Kong, consumed after a main meal, similar to the way dessert would be consumed in Western cultures, may also contain high FODMAP ingredients, and these include dried longan, dried dates and mango. Other sweet soups common to China and Hong Kong contain high FODMAP legumes, in particular, "red been sweet soup," and "green (mung) bean soup." Soya, red and mung beans are also incorporated into savoury dishes, such as "zongzi," triangular glutinous rice packages wrapped in a lotus/bamboo leaf and in vegetarian meals. Another protein source used in vegetarian dishes is "mock" meat. Although classification of FODMAP content in mock meat has not been fully completed, "vegetarian mince" has recently been tested and classified as high in FODMAPs (fructans/GOS,) and therefore, further analyses of mock meats are required to understand what role they may play in managing patients' IBS symptoms.

In addition to its Chinese heritage, Hong Kong is also influenced by Western culture due to its period under British rule between 1841 and 1997. Consequently, the cuisine in Hong Kong ranges from deeply traditional Chinese dishes and meals frequently served in "cha cha tengs," the equivalent of local cafes, to a fusion of foods between British and Chinese cuisines. This includes French toast (made from wheat) with butter and sweetened condensed milk (lactose-containing) or soupy macaroni (wheat-based pasta) and ham dishes that are commonly consumed for breakfast. While several key high FODMAP-containing ingredients are found in Chinese dishes, usual serving sizes of each need to be clearly defined and analysed before meals/condiments can be given a definite FODMAP rating.

\section{Taiwanese Dishes}

Taiwanese cuisine borrows heavily from Chinese cuisine due to successive waves of migration from China triggered by social unrest and war on the mainland, but it retains a distinct identity due to its isolation island locale. Its cuisine generally consists of the same high FODMAP containing vegetables as in China and Hong Kong. Taiwanese street food is world renowned for "stinky tofu," the poster child of Taiwanese street food. This is likely to be high in FODMAPs due to the sauce/condiments that accompany the tofu such as onion, garlic, gochujang (as noted in Korean dishes) and broccoli. Another quintessential street food is "youtiao" (deep fried dough sticks), which are made with wheat flour. Essential elements of the Taiwanese pantry include chilli bean sauce (made from fermented black soy beans or yellow fava beans), dried shitake mushrooms, fermented black beans, "sha-cha" sauce (made with dried shrimp, fish, shallots, garlic, and dried chillies) and fried shallots. Sha-cha sauce may then be added to soups, used as a marinade for meats or as a seasoning to stir fries. Finally desserts and drinks in Taiwan are frequently made with milk. In particular the popular "crushed iced-milk drink," is made with either whole milk or sweetened condensed milk, and served with fruit. This is similar to "smoothies" or "milkshakes" that are common in Western cultures. The likelihood that these dessert-type drinks being classified as a high FODMAP beverage/food is a reasonable assumption to make given milk (lactosecontaining) is a key and major ingredient.

\section{Vietnamese Dishes}

Vietnamese cuisine, heavily influenced by the Chinese and French, consists of traditional side condiments or adjuncts to most dishes. Some of these include "Hanh phi" (fried shallots in vegetable oil), "Toi phi va mo toi" (fried garlic) and "Nuoc man cham" (dipping fish sauce). The fish sauce, which has a sweet and sour spicy flavor, includes ingredients such as vinegar, sugar, chilli, lime juice and garlic. This sauce is added to many dishes, particularly to traditional Vietnamese rice vermicelli salads, which are then garnished with fried shallots and nuts. Rice vermicelli salads may also include grilled meats that are marinated in several ingredients, some containing a high content of FODMAPs, particularly scallions, honey, and the dipping fish sauce.

Another well-known popular traditional Vietnamese cuisine is "Pho bo tai nam" (beef noodle soup), simply referred to as "Pho." A key ingredient to this dish is white onion, a high FODMAPcontaining ingredient, which is finely sliced into rings and added fresh to the soup. The broth for Pho also contains a significant amount of onions. Although the liquid component of the broth is separated from bulky ingredients, which are discarded before use, it is important to note that oligosaccharides (present in onions) are water soluble and, therefore, likely to leak into the liquid component of the stock. Large batches of "Nuoc leo ga" (chicken stock) is also popular and traditionally made with an abundance of high FODMAP ingredients such as garlic and the white part of scallions. This is then stored in large quantities due to its frequent use in dishes for subsequent use. Thus, Pho and Nuco leo ga are likely to have a high concentration of FODMAPs and, because they are consumed in relatively large volumes, a serving size will be a high FODMAP food. Finally, due to the French influence, wheat bread (fructan-containing) is a common feature of Vietnamese cuisine, such as "Vietnamese pork rolls" or as an adjunct to Vietnamese curries. 


\section{Conclusion}

IBS is a global problem that affects one in 10 people in most populations. It impairs quality of life, has large economic costs and is challenging to manage for health professionals and patients alike. Efficacious dietary strategies have the advantage of providing a means of successful self-management, reducing the need for expensive pharmacological and complementary medicines. The emergence of quality data on the efficacy of the low FODMAP diet, and its widespread application and apparent success across Western countries has indeed placed this within first-line therapies for IBS in many centres. There is no reason why it will not be similarly efficacious in East and Southeast Asian communities visceral hypersensitivity is believed to be the major underlying pathophysiology abnormality and high FODMAP food ingredients make up a considerable proportion of most cuisines, as illustrated above. However, implementation of the diet has more uncertainties in East and Southeast Asian countries due to a limited database of FODMAP content of ingredients, to the uncertain effects of preparation on FODMAP content, and to the content per standard serve size of the final food that is eaten. There has also been little exploration of other FODMAP groups less relevant in Western cuisine, such as IMO, given the frequent use of fermented soy bean and other products. Thus, the great promise this dietary approach holds for treating this common condition in many Asian countries needs to be underpinned with further food composition studies and research into its efficacy among patient populations within East and Southeast Asia.

\section{References}

1. Canavan C, West J, Card T. Review article: the economic impact of the irritable bowel syndrome. Aliment Pharmacol Ther 2014;40: 1023-1034.

2. Nam KC, Jo C, Lee M. Meat products and consumption culture in the East. Meat Sci 2010;86:95-102.

3. Lovell RM, Ford AC. Global prevalence of and risk factors for irritable bowel syndrome: a meta-analysis. Clin Gastroenterol Hepatol 2012;10:712-721, e4.

4. Kim M, Park H. The process of symptom control in korean women with irritable bowel syndrome. Gastroenterol Nurs 2011;34:424-432.

5. Han SH, Lee OY, Bae SC, et al. Prevalence of irritable bowel syndrome in Korea: population-based survey using the Rome II criteria. J Gastroenterol Hepatol 2006;21:1687-1692.

6. Gwee KA, Bak YT, Ghoshal UC, et al. Asian consensus on irritable bowel syndrome. J Gastroenterol Hepatol 2010;25:1189-1205.

7. Lee YY, Waid A, Tan HJ, Chua AS, Whitehead WE. Rome III survey of irritable bowel syndrome among ethnic Malays. World J Gastroenterol 2012;18:6475-6480.

8. Gwee KA, Lu CL, Ghoshal UC. Epidemiology of irritable bowel syndrome in Asia: something old, something new, something borrowed. J Gastroenterol Hepatol 2009;24:1601-1607.

9. Ong DK, Mitchell SB, Barrett JS, et al. Manipulation of dietary short chain carbohydrates alters the pattern of gas production and genesis of symptoms in irritable bowel syndrome. J Gastroenterol Hepatol 2010;25:1366-1373.

10. Halmos EP, Power VA, Shepherd SJ, Gibson PR, Muir JG. A diet low in FODMAPs reduces symptoms of irritable bowel syndrome. Gastroenterology 2014;146:67-75, e5.

11. Staudacher HM, Whelan K, Irving PM, Lomer MC. Comparison of symptom response following advice for a diet low in fermentable carbohydrates (FODMAPs) versus standard dietary advice in patients with irritable bowel syndrome. J Hum Nutr Diet 2011;24: 487-495.

12. Staudacher HM, Lomer MC, Anderson JL, et al. Fermentable carbohydrate restriction reduces luminal bifidobacteria and gastrointestinal symptoms in patients with irritable bowel syndrome. J Nutr 2012;142:1510-1518.

13. Tuck CJ, Muir JG, Barrett JS, Gibson PR. Fermentable oligosaccharides, disaccharides, monosaccharides and polyols: role in irritable bowel syndrome. Expert Rev Gastroenterol Hepatol 2014;8: 819-834.

14. Chen HL, Lu YH, Lin JJ, Ko LY. Effects of isomalto-oligosaccharides on bowel functions and indicators of nutritional status in constipated elderly men. J Am Coll Nutr 2001;20:44-49.

15. Kohmoto T, Tsuji K, Kaneko T, et al. Metabolism of ${ }^{13} \mathrm{C}$-isomaltooligosaccharides in healthy men. Biosci Biotechnol Biochem 1992;56: 937-940.

16. White Jr JW, Hoban N. Composition of honey. IV. Identification of the disaccharides. Arch Biochem Biophys 1959;80:386-392.

17. Murray K, Wilkinson-Smith V, Hoad C, et al. Differential effects of FODMAPs (fermentable oligo-, di-, mono-saccharides and polyols) on small and large intestinal contents in healthy subjects shown by MRI. Am J Gastroenterol 2014;109:110-119.

18. Marciani L, Cox EF, Hoad CL, et al. Postprandial changes in small bowel water content in healthy subjects and patients with irritable bowel syndrome. Gastroenterology 2010;138:469-477, e1.

19. Barrett JS, Ng PS, Muir JG, Gibson PR. Letter: oral fructose breath hydrogen response, symptoms, both or neither? Aliment Pharmacol Ther 2013;38:442-443.

20. Gibson GR, Probert HM, Van Loo J, Rastall RA, Roberfroid MB. Dietary modulation of the human colonic microbiota: updating the concept of prebiotics. Nut Res Rev 2004;17:259-275.

21. D'Argenio G, Cosenza V, Delle Cave M, et al. Butyrate enemas in experimental colitis and protection against large bowel cancer in a rat model. Gastroenterology 1996;110:1727-1734.

22. Jain I, Kumar V, Satyanarayana T. Xylooligosaccharides: an economical prebiotic from agroresidues and their health benefits. Indian J Exp Biol 2015;53:131-142.

23. Antalis TM, Reeder JA. Butyrate regulates gene expression of the plasminogen activating system in colon cancer cells. Int $\mathrm{J}$ Cancer 1995;62:619-626.

24. Halmos EP, Christophersen CT, Bird AR, Shepherd SJ, Gibson 
PR, Muir JG. Diets that differ in their FODMAP content alter the colonic luminal microenvironment. Gut 2014;64:93-100.

25. Whigham L, Joyce T, Harper G, et al. Clinical effectiveness and economic costs of group versus one-to-one education for short-chain fermentable carbohydrate restriction (low FODMAP diet) in the management of irritable bowel syndrome. J Hum Nutr Diet Published Onlie First: 14 Apr 2015. doi: 10.1111/jhn.12318.

26. Monash University, Department of Gastroenterology. The Monash University low FODMAP diet app. Melbourne Australia: Central Clinical School 2015 [updated 28 May 2015; cited 20154 June]. Available from URL: http://www.med.monash.edu.au/cecs/gastro/ fodmap/iphone-app.html (accessed 9 Sep 2015).

27. Monash University. The Monash University Low FODMAP diet booklet. 5 ed. Melbourne, Australia: Department of Gastroenterology, Central Clinical School 2015. Available from URL: http://ecommerce. med.monash.edu.au/product.asp?pID $=317 \& c I D=11$ (accessed 9 Sep 2015).

28. Marsh A, Eslick EM, Eslick GD. Does a diet low in FODMAPs reduce symptoms associated with functional gastrointestinal disorders? A comprehensive systematic review and meta-analysis. Eur J Nutr Published Online First: 17 May 2015. doi: 10.1007/s00394015-0922-1.

29. Biesiekierski JR, Peters SL, Newnham ED, Rosella O, Muir JG, Gibson PR. No effects of gluten in patients with self-reported non-celiac gluten sensitivity after dietary reduction of fermentable, poorly absorbed, short-chain carbohydrates. Gastroenterology 2013; 145:320-328, e1-e3.

30. Chumpitazi BP, Cope JL, Hollister EB, et al. Randomised clincial trial: gut microbiome biomarkers are associated with clinical response to a ow FODMAP diet in children with irritable bowel syndrome. Aliment Pharmacol Ther 2015;42:418-427.
31. De Roest RH, Dobbs BR, Chapman BA, et al. The low FODMAP diet improves gastrointestinal symptoms in patients with irritable bowel syndrome: a prospective study. Int J Clin Pract 2013;67: 895-903.

32. American College of Gastroenterology Task Force on Irritable Bowel Syndrome, Brandt LJ, Chey WD, et al. An evidence-based position statement on the management of irritable bowel syndrome. Am J Gastroenterol 2008;104(suppl 1):S1-S35.

33. Heizer WD, Southern S, McGovern S. The role of diet in symptoms of irritable bowel syndrome in adults: a narrative review. J Am Diet Assoc 2009;109:1204-1214.

34. Halpert A, Dalton CB, Palsson O, et al. What patients know about irritable bowel syndrome (IBS) and what they would like to know. National survey on patient educational needs in IBS and development and validation of the patient educational needs questionnaire (PEQ). Am J Gastroenterol 2007;102:1972-1982.

35. Muir JG, Rose R, Rosella O, et al. Measurement of short-chain carbohydrates in common Australian vegetables and fruits by high-performance liquid chromatography (HPLC). J Agric Food Chem 2009;57:554-565.

36. Muir JG, Shepherd SJ, Rosella O, Rose M, Barrett JS, Gibson PR. Fructan and free fructose content of common Australian vegetables and fruit. J Agric Food Chem 2007;55:6619-6627.

37. Pedersen N, Andersen NN, Végh Z, et al. Ehealth: low FODMAP diet vs Lactobacillus rhamnosus GG in irritable bowel syndrome. World J Gastroenterol 2014;20:16215-16226.

38. Pedersen N, Vegh Z, Burisch J, et al. Ehealth monitoring in irritable bowel syndrome patients treated with low fermentable oligo-, di-, mono-saccharides and polyols diet. World J Gastroenterol 2014;20: 6680-6684. 